\title{
Collaborative coding and decoding techniques for multiple access channel
}

\author{
F. Ali \\ B. Honary, MIEE, CEng
}

Indexing terms: Coding, Decoding, Multiple access channel, Error control

\begin{abstract}
It is highly desirable to use simple and effective multiple access coding and decoding techniques which are capable of multiple access function and error control. The collaborative coding multiple access (CCMA) techniques potentially permit efficient simultaneous transmission by several users sharing a common channel, without subdivision in time, frequency or orthogonal codes. The authors investigate the performance of uniquely decodable CCMA schemes employing hard decision and maximum likelihood decoding techniques. A low complexity maximum likelihood decoding technique is presented. The reliability performance of various coding schemes employing these decoding techniques are carried out in the presence of AWGN conditions. The simulation results are presented in the form of symbol and codeword error rates as a function of signal to noise ratios. It is shown that uniquely decodable CCMA schemes permit the multiple access function to be combined with that of forward error correction.
\end{abstract}

\section{Introduction}

The multiple access channel (MAC) communication system is depicted in Fig. 1 , in which there are $T$ independent sources transmitting data to $T$ separate destinations over a common discrete channel with one decoder serving $T$ sinks. The inputs and their associated sources and encoders may be in different physical locations; for example, different rooms in a building or different mobiles in an area. The signals over the channel will interfere, superimpose or combine in some way. The single decoder at the receiver is required to unscramble and deliver the messages to their corresponding sinks, if possible, without errors. In the collaborative coding schemes, the $T$ messages generated from the $T$ sources are encoded independently such that they are interference free during simultaneous transmission over a common channel. Each user is provided with a code which enables the receiver to unscramble the individual information

\section{(C) IEE, 1994}

Paper 1043I (E5, E7), first received 21st April and in revised form 29th November 1993

F. Ali was with, and B. Honary is with the Hull-Lancaster Communications Research Group, Engineering Department, Lancaster University, Lancaster LA1 4YR, United Kingdom

F. Ali is now with the School of Engineering, University of Sussex, Falmer, Brighton BN1 9QT, United Kingdom streams, by detecting the resulting combined signal. The information theory of MAC communications was initiated in 1961 by Shannon in his fundamental paper [1],

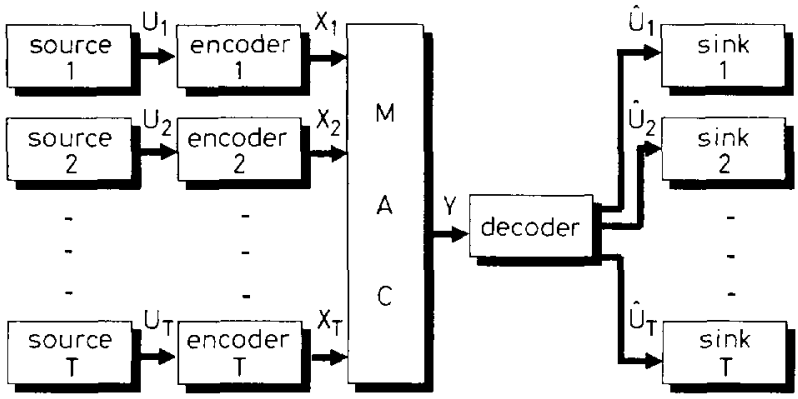

Fig. 1 Block diagram of T-user multiple access channel

and established in 1971 with a coding theorem developed in References 2 and 3. Surveys of the information theory approach are given in References 4-7 and various coding constructions for the $T$-user MAC are given in References 8-21.

In the following section of this paper, $T$-user CCMA techniques are described. Section 3 describes hard decision decoding of CCMA techniques. A low complexity maximum likelihood decoding technique is presented in Section 4 to utilise the error control capability. The generalised decoding procedure and algorithm are given. A particular two-user uniquely decodable scheme is taken as an example and analysed with this technique. The error probability analysis is given in Section 5 . The reliability of various coding schemes employing hard decision and maximum likelihood decoding techniques are carried out by simulation in the presence of AWGN conditions. The simulation results and discussions are given in Section 6.

\section{T-user CCMA schemes}

In Fig. 1, data from the $i$ th source, $U_{i}$, where $i=1,2, \ldots$, $T$, is encoded by the $i$ th encoder according to a uniquely assigned block code $C_{i}$ of length $N$. The resulting codeword vector $X_{i}$ is then transmitted over the channel where it combines with the other $(T-1)$ codeword vectors to produce a composite codeword vector $Y$ of length $N$. The transmitters are assumed to operate in perfect symbol and block synchronisation over a common discrete $T$-user MAC. At the receiving end the single decoder decodes $Y$ into estimates of the original data streams $U_{1}, U_{2}, \ldots, U_{T}$.

The $T$ codes $C_{1}, C_{2}, \ldots, C_{T}$ together are called a ' $T$-user collaborative code', where each component is termed a 'constituent code'. If all the constituent codes are binary block codes then the codeword vector $X_{i}$ is an 
$N$-symbol binary vector. The transmission rate (in bits per channel use) of the $i$ th constituent code $C_{i}$ containing $C W_{i}$ codewords each of length $N$ and each codeword equally likely, is given by

$$
R_{i}=\log _{2}\left(C W_{i}\right) / N
$$

and the rate sum, $R_{\text {sum }}$, of all the users is given by

$$
R_{\text {sum }}=\sum_{i=1}^{T} \log _{2}\left(C W_{i}\right) / N
$$

Various block collaborative coding schemes have been constructed for different number of users $(T \geqslant 2)$ over the years for noiseless and noisy MACs [8-21]. These constructions have followed various approaches to achieve the bounds promised by the multiple access information theory. As an example, a simple coding scheme for a twouser uniquely decodable code with block length of $N=2$, is given here [8-11]. The codewords for user 1 and 2 are $C_{1}=(00,11)$ and $C_{2}=(00,01,10)$, respectively. This twouser code $\left(C_{1}, C_{2}\right)$ is uniquely decodable because all the received composite codewords are distinct as shown in Table 1. Therefore the decoder can unscramble the two messages without any ambiguity. Generally, in noiseless channel conditions, the decoder is capable of decoding every received composite codeword vector, without ambiguity, into $T$ codewords that were transmitted by the $T$ encoders. However, if the channel is noisy, the decoder chooses the codeword which is closest to the received, as measured by some metric distance.

Table 1 : Two-user uniquely decodable code

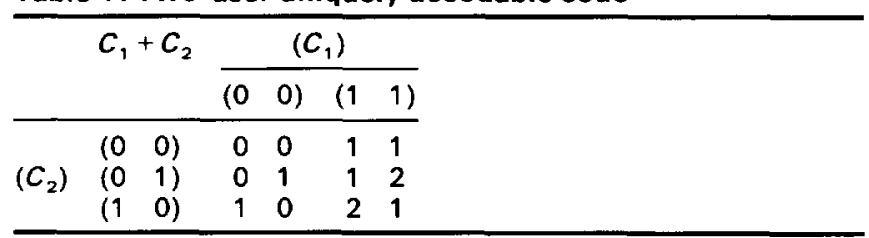

Uniquely decodable coding schemes can also have some error protection capability [8-21]. In particular, codes have been found for the two-user binary adder MAC with rates up to 1.292 bits per channel use which achieve the MAC function and offer some error protection capability. The decoding of $T$-user collaborative coding schemes is based here on two techniques, hard and soft decision decoding.

\section{$3 \quad$ Hard decision (HD) decoding}

In HD decoding of CCMA schemes the demodulator sets $(T)$ decision thresholds to detect the $(T+1)$ possible signal levels transmitted by the $T$-user. Here, each received symbol is detected independently for $N$ received symbols, hence is called symbol-by-symbol HD (SBS_HD) decoding. However, this decoding technique cannot be used on its own to perform the full decoding process to deliver the individual users information to their intended destinations. This is due to the fact that sometimes in noisy conditions the SBS_HD decoding results in a codeword which is not admissible. In this case the decoder will fail to deliver the individual users information. Therefore $L$-distance $H D$ decoding is used in conjunction with SBS_HD to complete the decoding process and resolve this ambiguity. This complete process is referred to as the HD_CCMA decoding technique. The HD_CCMA decoder calculates all the $L$-distances between the SBS_HD codeword and all the possible admissible codewords. Then, the codeword with the least $L$-distance is chosen. The $L$-distance between two $N$ symbol composite codewords $Z$ and $Z^{\prime}\left(Z \neq Z^{\prime}\right)$ is defined as follows:

$$
d_{L}\left(Z, Z^{\prime}\right)=\sum_{i=1}^{N}\left|z_{i}-z_{i}^{\prime}\right|=\left\|Z-Z^{\prime}\right\|
$$

where the minus sign denotes real subtraction, $\left|z_{i}-z_{i}^{\prime}\right|$ denotes the absolute value of $z_{i}-z_{i}^{\prime}$ and the symbol $\left\|Z-Z^{\prime}\right\|$ means that the $L$-distance is a metric. This kind of decoding guarantees correct decoding in the noiseless uniquely decodable coding scheme. However, in the noisy case the number of errors which can be corrected under this decoding is $t=\left\lfloor\left(d_{\min }-1\right) / 2\right\rfloor$, where $d_{\min }$ is the minimum $L$-distance of a $T$-user collaborative code, which is the smallest value of $d_{L}\left(Z, Z^{\prime}\right)$ over all $Z \neq Z^{\prime}$, and $[x]$ means an integer number less than or equal to $x$.

\section{Maximum likelihood soft decision (MLSD) decoding}

Consider a set of composite codewords each comprising $N$ symbols and assume the received signal is $W=$ $\left(w_{1}, w_{2}, \ldots, w_{N}\right)$, where $w_{i}$ is the magnitude of the element representing the $i$ th symbol. In principle, the joint maximum likelihood (ML) decision carried out on the complete word is a very powerful detection technique. Therefore, if the actual signal magnitude of the $N$-symbol codeword is made available to the decoder, then an ML decoding for CCMA schemes can be performed. This is achieved by calculating the Euclidean distances between the received codeword and all the admissible codewords. The codeword with minimum Euclidean distance (MED) is chosen as the received codeword. Provided the codewords are all equally likely, this strategy is optimum in the sense that it minimises the probability of error in the decoder.

However, this technique is difficult to implement in practice, because this would require the storage of the precise amplitudes of all symbols as received. In addition, the decoding table becomes unmanageably large as the length of the code and the number of active users increases. Therefore, what is needed is a simple means of calculating the possible transmitted codewords from the received codeword with the least number of operations possible.

Here, a low complexity ML decoding technique is introduced. This technique has the reliability of ML decoding with less implementation complexity and reduces the number of computations required to decode a received codeword [22]. The decoding problem at the receiver can be defined as follows: "given $W=\left(w_{1}, w_{2}\right.$, $\left.\ldots, w_{N}\right)$, where $w_{i}$ is a real valued scalar, it is necessary to decode the transmitted codeword in such a way that the total probability of codeword error is minimised'. For a given $T$-user CCMA code structure, two sets of 'admissible' and 'forbidden' codewords are defined. Assume $A_{i}=\left(a_{i 1}, a_{i 2}, \ldots, a_{i N}\right)$ is the $i$ th admissible codeword where $i=1,2, \ldots, N_{a} ; N_{a}$ is the number of admissible codewords given by

$$
N_{a}=\prod_{l=1}^{T} C W_{l}
$$

$C W_{l}$ is the number of codewords in the $l$ th user, and $a_{i j}$ is the $j$ th symbol value of the $i$ th admissible codeword where $j=1,2, \ldots, N$. A set of forbidden codewords for certain error conditions is defined as $F_{i}=$ $\left(f_{i 1}, f_{i 2}, \ldots, f_{i N}\right)$, where $i=1,2, \ldots, N_{f} ; N_{f}$ is the number 
of forbidden codewords, and $f_{i j}$ is the $j$ th symbol value of the $i$ th forbidden codeword where $j=1,2, \ldots, N$.

If we asume the transmitted codeword is $A_{k}=\left(a_{k 1}\right.$, $\left.a_{k 2}, \ldots, a_{k N}\right)$, and the received codeword is $W=\left(w_{1}, w_{2}\right.$, $\left.\ldots, w_{N}\right)$. Then, in order to construct the decision decoding table to decode the received codeword, the following procedure is required:

(i) Define the subset of admissible codewords nearest to each forbidden codeword for certain error conditions.

(ii) Calculate all the Euclidean distances between the received codeword $W=\left(w_{1}, w_{2}, \ldots, w_{N}\right)$ and all the codewords from the admissible codewords subset nearest to the $i$ th forbidden codeword.

(iii) Choose the codeword with the MED. That is, if the generalised distance between $W$ and the admissible codeword, say $A_{p} \in\left(A_{i}\right)$ is minimum, then $A_{p}=\left(a_{p 1}, a_{p 2}\right.$, $\left.\ldots, a_{p N}\right)$ is accepted as the transmitted codeword.

(iv) Comparison thresholds are then found for each forbidden codeword to form the decoding table for this decoding technique.

This decoding technique is also used in conjunction with SBS_HD decoding. This arrangement allows one to correct some errors which cannot be corrected using SBS_HD and HD_CCMA decoding techniques. In addition this decoding scheme may perform both error detection and correction at the same time.

\subsection{Decoding algorithm}

The generalised decoding algorithm steps for this MLSD CCMA decoding technique can be summarised as follows:

Step 1: Perform SBS_HD decoding on the received codeword $W=\left(w_{1}, w_{2}, \ldots, w_{N}\right)$.

Step 2: 'Error detection': perform error detection by checking the SBS_HD decoded codeword, whether it is admissible or forbidden; if it is admissible go to step 4, else continue.

Step 3: 'Error correction': perform error correction by checking the decision thresholds (calculated for a given $T$-user coding scheme as shown in the procedure above in Section 4) stored in the decoding decision table for the current forbidden codeword.

Step 4: Individual users' information is then decoded by using the normal decoding procedure used in the noiseless case.

It can be seen from the above steps that it is not necessary to calculate the Euclidean distances between the received codeword and all the admissible codewords every time a codeword is received. It is only necessary to check certain conditions according to a decision decoding table. Therefore, in addition to performing MLSD decoding, the total number of operations are reduced compared to conventional techniques.

\subsection{Example: two-user coding scheme}

As an example and for analysis of this MLSD_CCMA decoding technique, the two-user code given in Table 1 is considered and referred to as code 1 . That is, user 1 and user 2 codewords are $C_{1}=(00,11), C_{2}=(00,01,10)$, respectively. The set of admissible composite codewords is $(00,10,01,11,12,21)$. The single error conditions for this coding scheme are defined as follows: if the transmitted symbols from each user are $(0,0)$ or $(1,1)$ and the received composite symbol is 1 ; and if $(0,1)$ or $(1,0)$ are transmitted and either 0 or 2 is received, then a single error has occurred during transmission. Therefore, for this single error condition, the set of forbidden composite code- words is $(02,20,22)$ and the subset of admissible composite codewords nearest to each forbidden codeword are defined as shown in Table 2.

Table 2: Forbidden and nearest admissible codewords for two-user code 1

\begin{tabular}{ll}
$\begin{array}{l}\text { Forbidden } \\
\text { codewords }\end{array}$ & $\begin{array}{l}\text { Nearest admissible } \\
\text { codewords }\end{array}$ \\
\hline 02 & $\longrightarrow$ \\
20 & $\longrightarrow(12,01)$ \\
22 & $\longrightarrow(21,10)$ \\
\hline
\end{tabular}

The construction of the decoding decision table can be obtained by calculating the Euclidean distances between the received codeword $W=\left(w_{1}, w_{2}\right)$ of a forbidden codeword and the nearest admissible codewords (defined in Table 2) as shown below:

(i) The Euclidean distances for the forbidden codeword 02:

$$
\begin{aligned}
d_{1}^{2}= & \left(w_{1}-1\right)^{2}+\left(w_{2}-2\right)^{2} ; \\
& \text { between } W=\left(w_{1}, w_{2}\right) \text { and } 12, \\
d_{2}^{2}= & \left(w_{1}-0\right)^{2}+\left(w_{2}-1\right)^{2} ; \\
& \text { between } W=\left(w_{1}, w_{2}\right) \text { and } 01, \\
d_{1}^{2}- & d_{2}^{2}=-2 w_{1}-2 w_{2}+4 \\
\text { then } d_{1}^{2}< & d_{2}^{2} \text { if } w_{1}+w_{2}>2
\end{aligned}
$$
therefore $d_{1}^{2}$ is the minimum distance.

(ii) The Euclidean distances for the forbidden codeword 20:

$$
\begin{aligned}
d_{3}^{2}= & \left(w_{1}-2\right)^{2}+\left(w_{2}-1\right)^{2} ; \\
& \text { between } W=\left(w_{1}, w_{2}\right) \text { and } 21, \\
d_{4}^{2}= & \left(w_{1}-1\right)^{2}+\left(w_{2}-0\right)^{2} ; \\
& \text { between } W=\left(w_{1}, w_{2}\right) \text { and } 10 \\
d_{3}^{2}- & d_{4}^{2}=-2 w_{1}-2 w_{2}+4 ; \\
\text { then } d_{4}^{2}> & d_{3}^{2} \text { if } w_{1}+w_{2}>2 ; \\
& \text { therefore } d_{3}^{2} \text { is the minimum distance. }
\end{aligned}
$$

(iii) The Euclidean distances for the forbidden codeword 22:

$$
\begin{aligned}
d_{5}^{2}= & \left(w_{1}-2\right)^{2}+\left(w_{2}-2\right)^{2} ; \\
& \text { between } W=\left(w_{1}, w_{2}\right) \text { and } 21, \\
d_{6}^{2}= & \left(w_{1}-1\right)^{2}+\left(w_{2}-2\right)^{2} ; \\
& \text { between } W=\left(w_{1}, w_{2}\right) \text { and } 12, \\
d_{5}^{2}- & d_{6}^{2}=-2 w_{1}+2 w_{2} ; \\
\text { then } d_{5}^{2}< & d_{6}^{2} \text { if } w_{1}>w_{2} ; \\
& \text { therefore } d_{5}^{2} \text { is the minimum distance }
\end{aligned}
$$

Therefore a decision table is constructed based on these calculations, to be used for the decoding purposes, as shown in Table 3.

Table 3: Decoding decision table for two-user code 1

\begin{tabular}{lll}
\hline $\begin{array}{l}\text { Forbidden } \\
\text { codewords }\end{array}$ & $\begin{array}{l}\text { Comparison } \\
\text { thresholds }\end{array}$ & $\begin{array}{l}\text { Decoding } \\
\text { decision }\end{array}$ \\
\hline 02 & $w_{1}+w_{2}>2$ & 12 \\
& $w_{1}+w_{2}<2$ & 01 \\
20 & $w_{1}+w_{2}>2$ & 21 \\
& $w_{1}+w_{2}<2$ & 10 \\
22 & $w_{1}>w_{2}$ & 21 \\
& $w_{1}<w_{2}$ & 12 \\
\hline
\end{tabular}

Suppose the transmitted codeword is (10) and the received soft information codeword is $W=(1.6,0.3)$. Then, performing SBS_HD decoding, the decoded codeword will be (20); this means there is a single error from the transmitted composite codeword. Employing the HD_CCMA decoding technique will result in two possible codewords (10) and (21) which have the same minimum $L$-distance from the codeword (20). Therefore, 
the HD_CCMA decoder chooses either codeword with equal probability. If (10) is chosen then a single error has been corrected, however, if the codeword (21) is chosen then a double error has been introduced.

Now, employing the MLSD_CCMA decoding technique, since an error is detected by the forbidden codeword (20) and $\left(w_{1}+w_{2}\right)<2$, then the output of the decoder is the codeword (10) as can be seen from Table 3 . Thus, a single error has been corrected from the SBS_HD decoder or a double error from the HD_CCMA decoder (if the HD_CCMA decoder had chosen the codeword (21)). Therefore, by employing MLSD_CCMA decoding improvement in the decoding is possible, which allows some of the detected errors to be corrected. This decoding technique will give higher improvement gain if the code used has some error protection capability.

\section{Error probability of MLSD_CCMA decoding}

The performance of the T-user MLSD_CCMA decoding scheme is evaluated here in terms of the probability of composite codeword error. The reliability of individual user's sink information depends on how accurate the composite codeword is decoded. These analyses are carried out over an AWGN channel of zero mean and variance $\sigma_{N}^{2}$. If the total number of admissible composite codewords for a given $T$-user code is $N_{a}$, then the total probability of correct decision can be written as

$$
P_{c c}(t o)=\sum_{i=1}^{N_{a}} p(i) P_{c c}(i)
$$

where $p(i)$ is the probability of the $i$ th admissible codeword,

$$
\sum_{i=1}^{N_{a}} p(i)=1
$$

and $P_{c c}(i)$ is the probability of correct decision on the $i$ th admissible codeword,

$$
P_{c c}(i)=\iint_{\left\{G_{i}\right\}} \cdots \int f_{i}\left(w_{1} w_{2} \cdots w_{N}\right) d w_{1} d w_{2} \cdots d w_{N}
$$

where $\left\{G_{i}\right\}$ is the region of correct decision of the ith composite codeword, and $f_{i}\left(w_{1} w_{2} \cdots w_{N}\right)$ is the joint PDF of the $i$ th admissible codeword, which can be written for the AWGN as

$$
\begin{aligned}
& f_{i}\left(w_{1} w_{2} \cdots w_{N}\right) \\
& \quad=\left[\frac{1}{\sqrt{ }(2 \pi) \sigma_{N}}\right]^{N} \exp \left\{\sum_{j=1}^{N}\left[\frac{-\left(w_{j}-a_{i j}\right)^{2}}{2 \sigma_{N}^{2}}\right]\right\}
\end{aligned}
$$

where $a_{i j}$ is the $j$ th symbol in the $i$ th admissible codeword, where $j=1,2, \ldots, N$ and $i=1,2, \ldots, N_{a}$. Substituting back into eqn. 7 and 5 , the total probability of correct decision is

$$
\begin{aligned}
P_{c c}(t o)= & \sum_{i=1}^{N_{a}}\left\langle p(i)\left[\frac{1}{\sqrt{(2 \pi) \sigma_{N}}}\right]^{N}\right. \\
& \left.\times \iint_{\left\{G_{i}\right.} \cdots \int \exp \left\{\sum_{j=1}^{N}\left[\frac{-\left(w_{j}-a_{i j}\right)^{2}}{2 \sigma_{N}^{2}}\right]\right\}\right\rangle \\
& \times d w_{1} d w_{2} \cdots d w_{N}
\end{aligned}
$$

and the total probability of error can be written as

$$
\begin{aligned}
P_{c c}(t o)= & 1-\sum_{i=1}^{N_{a}}\left\langle p(i)\left[\frac{1}{\sqrt{(2 \pi) \sigma_{N}}}\right]^{N}\right. \\
& \left.\times \iint_{\left\{G_{i j}\right.} \cdots \int \exp \left\{\sum_{j=1}^{N}\left[\frac{-\left(w_{j}-a_{i j}\right)^{2}}{2 \sigma_{N}^{2}}\right]\right\}\right\rangle \\
& \times d w_{1} d w_{2} \cdots d w_{N}
\end{aligned}
$$

The region of correct decision $\left\{G_{i}\right\}$ represent the set of points for which the Euclidean distance to the $i$ th admissible codeword is smaller than for all other admissible codewords. That is :

$$
\left\{G_{i}\right\}=\left\{\left(w_{1} w_{2} \cdots w_{N}\right\}: \sum_{j=1}^{N}\left(w_{j}-a_{i j}\right)^{2}<\sum_{j=1}^{N}\left(w_{j}-a_{k j}\right)^{2}\right.
$$

where $\left(w_{1} w_{2} \cdots w_{N}\right)$ is the set of points in $\left\{G_{i}\right\}$; the colon sign (:) means defined as; $w_{j}$ are real-valued scalars which represent the coordinates for each point in the region $\left\{G_{i}\right\} ; a_{i j}$ and $a_{k j}$ are the $j$ th symbols in the $i$ th and $k$ th admissible codewords, respectively, where $i=1,2, \ldots$, $N_{a}, j=1,2, \ldots, N$, and $i \neq k$.

Similarly, this procedure can also be used to calculate the composite symbol and codeword error rate for CCMA schemes employing hard decision decoding techniques.

\section{$6 \quad$ Simulation results and discussions}

The simulation is carried out to evaluate the reliability performance of CCMA schemes employing various coding and decoding schemes. Various two-user CCMA schemes are introduced first and used throughout the simulation analysis. These collaborative codes are chosen to be simple short codes with summary rate, in most cases, higher than one bit/channel use. In addition, they are chosen to have different error protection capability of the overall two-user code and it's constituent codes. These codes are:

(a) Code 1: $C_{1}=(00,11), C_{2}=(00,01,10)$,

$C W_{1}=2, N_{1}=2, R_{1}=0.5, d_{1 \text { min }}=2$,

$C W_{2}=3, N_{2}=2, R_{2}=0.792, d_{2 \min }=1$,

$R_{\text {sum }}=1.292, d_{\min }=1$.

(b) Code 2: $C_{1}=(000,111)$,

$C_{2}=(000,001,010,011,100,101,110)$,

$C W_{1}=2, N_{1}=3, R_{1}=0.333, d_{1 \text { min }}=3$,

$C W_{2}=7, N_{2}=3, R_{2}=0.935, d_{2 \min }=1$,

$R_{\text {sum }}=1.269, d_{\min }=1$.

(c) Code 3: $C_{1}=(0000,0011,1100,1111)$,

$$
C_{2}=(0000,0001,0010,0100,0101,0110 \text {, }
$$

$C W_{1}=4, N_{1}=4, R_{1}=0.5, d_{1 \min }=2$,

$C W_{2}=9, N_{2}=4, R_{2}=0.792, d_{2 \min }=1$,

$R_{\text {sum }}=1.292, d_{\min }=1$.

(d) Code 4: $C_{1}=(0000,0001,0011,1100,1110,1111)$,

$C_{2}=(0000,0101,0110,1001,1010,1101)$,

$C W_{1}=6, N_{1}=4, R_{1}=0.646, d_{1 \min }=1$,

$C W_{2}=6, N_{2}=4, R_{2}=0.646, d_{2 \min }=1$,

$R_{\text {sum }}=1.292, d_{\min }=1$.

(e) Code 5: $C_{1}=(00,11), C_{2}=(10,01)$,

$$
\begin{aligned}
& C W_{1}=2, N_{1}=2, R_{1}=0.5, d_{1 \min }=2, \\
& C W_{2}=2, N_{2}=2, R_{2}=0.5, d_{2 \min }=2,
\end{aligned}
$$$$
R_{\text {sum }}=1.0, d_{\min }=2 \text {. }
$$

It is assumed that the two-user CCMA communication system is in perfect synchronisation. Modulation and 
demodulation are assumed to be available for these codes and considered to be part of the discrete channel. The channel is assumed to be AWGN of zero mean and variance $\sigma_{N}^{2}$. The ratio $E / N_{0}$, is defined here as the average signal energy per user to noise power spectral density given by $\sigma_{N}^{2}=N_{0} / 2$. The composite codeword error rate (CER) and the constituent users sink SER are calculated for each two-user collaborative code.

The composite CER versus $E / N_{0}$, employing HD_CCMA decoding is shown in Fig. 2, for all five

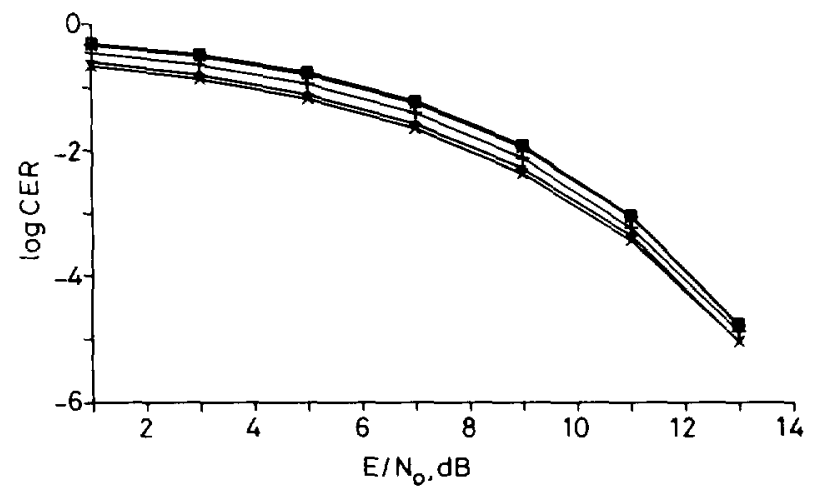

Fig. 2 HD_CCMA decoder CER

- - code 1

1- code 2

- 7 - code 4

$-x-\operatorname{code} 5$

codes. It can be seen from this figure that the reliability of these codes are very similar, since their protect capability is the same under HD_CCMA decoding. The small difference is due to the variation in the number of admissible and forbidden codewords from one code to another. The composite CER versus $E / N_{0}$ employing the MLSD_CCMA decoding is shown in Fig. 3 for all five

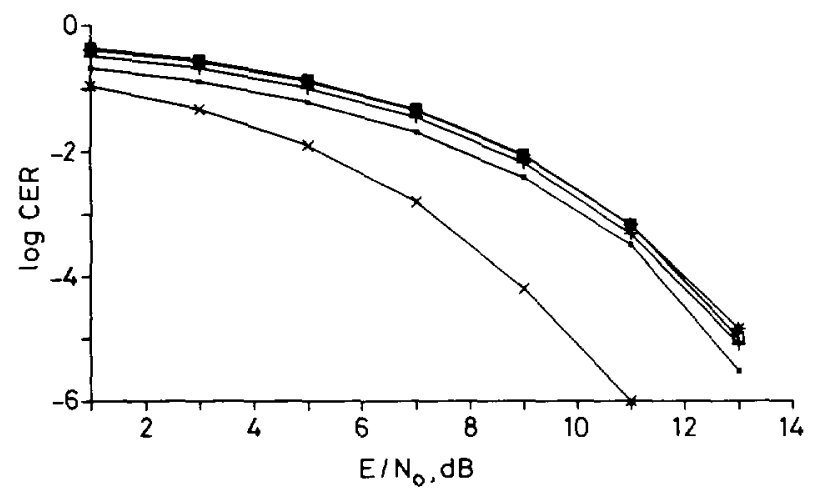

Fig. 3 MLSD_CCMA decoder CER

$\begin{array}{ll}- & \text { code } 1 \\ -1- & \text { code } 2 \\ -*- & \text { code } 3 \\ -\square- & \text { code } 4 \\ -x- & \text { code } 5\end{array}$

codes. It can be seen clearly that code 5 gives the best performance because its $d_{\min }=2$, which means that under this decoding a single error can be corrected.

For comparison purposes, and calculating the energy gain achieved by employing MLSD_CCMA decoding, the CER for codes 1 and 5 is presented separately in Figs. 4 and 5, respectively. Also included with these Figures is the CER for SBS_HD decoding. It can be seen from Fig. 4 that the MLSD_CCMA decoding gives better performance with some detection gain (because $d_{\min }=1$ for code 1). However, this gain is much higher when the code employed has some error correction capability $\left(d_{\min } \geqslant \neq\right.$ 2 ) as can be seen very clearly in Fig. 5. The gain achieved is more than $2.5 \mathrm{~dB}$ at an error probability of $10^{-6}$.

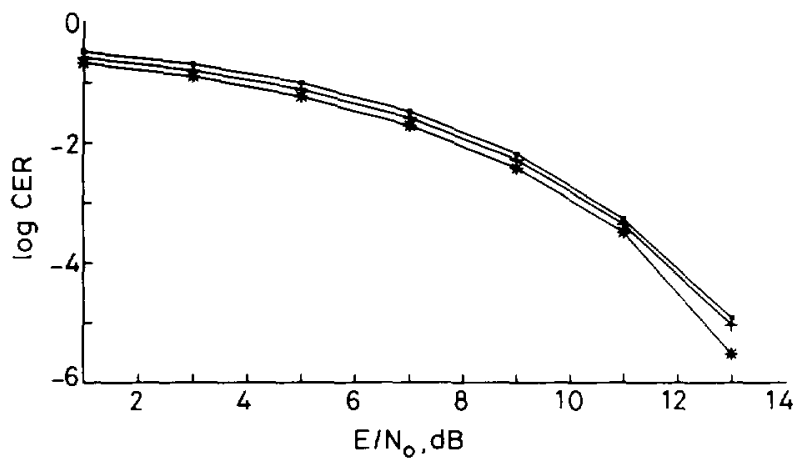

Fig. 4 CCMA decoding schemes CER (code 1)

- - SBS_HD

+ HD_CCMA

-

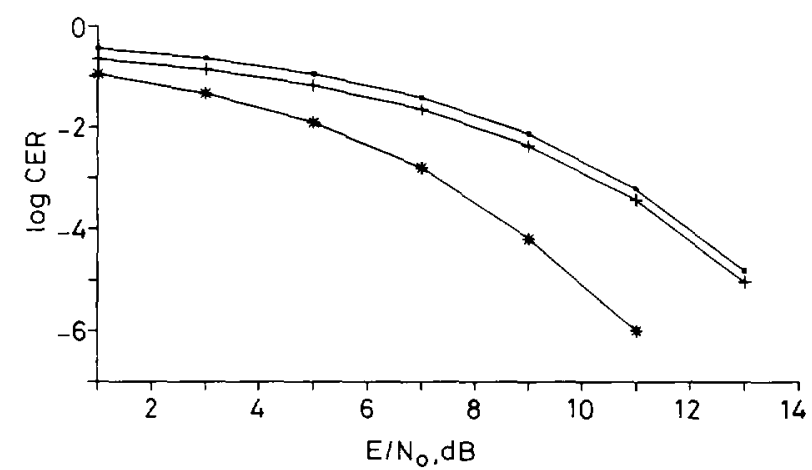

Fig. 5 CCMA decoding schemes CER (code 5)

$\begin{array}{ll}-1- & \text { SBS_HD } \\ -* \text { HD_CCMA } \\ -* \text { MLSD_CCMA }\end{array}$

The effect of employing these coding and decoding techniques is also investigated on the constituent codes and hence the user's sink data. The sink SER for each user is presented in Figs. 6-10 for all codes. It can be seen, for example in Fig. 6, that user 2 sink SER is very

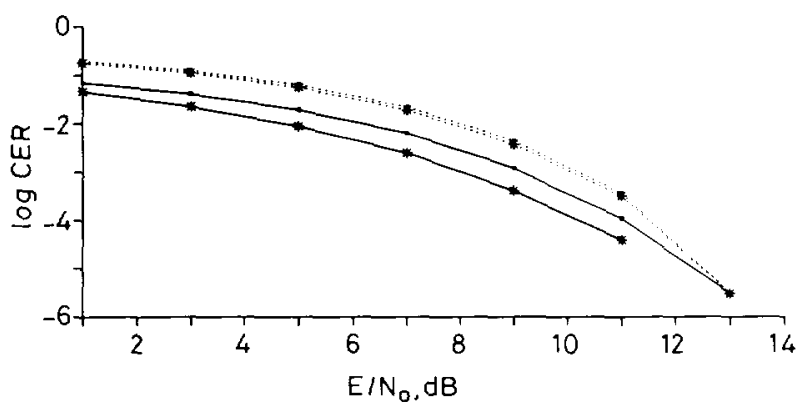

Fig. 6 CCM A decoding schemes users sink SER (code I)

$$
\begin{aligned}
& \text { - - User } 1 \text { HD_CCMA } \\
& \text { User } 1 \text { MLSD_CCMA } \\
& \text { User } 2 \text { HD_CCMA } \\
& \text { User } 2 \text { MLSD_CCMA }
\end{aligned}
$$

close for both MLSD_CCMA and HD_CCMA decoding techniques. However, user 1 reliability employing MLSD_CCMA decoding is better than HD_CCMA decoding because $d_{1 \min }=2$. This gain is also shown in Figs. 7 and 8 for user 1 of code 2 and code 3 , respectively. Since code 4 is a balanced code, the reliability of each user is very close as shown in Fig. 9. Code 5 is also a 
balanced code with $d_{1 \min }=d_{2 \min }=d_{\min }=2$. Therefore, the sink SER is the same for each user as shown in Fig. 10. It can also be seen from Fig. 10 that a coding gain of

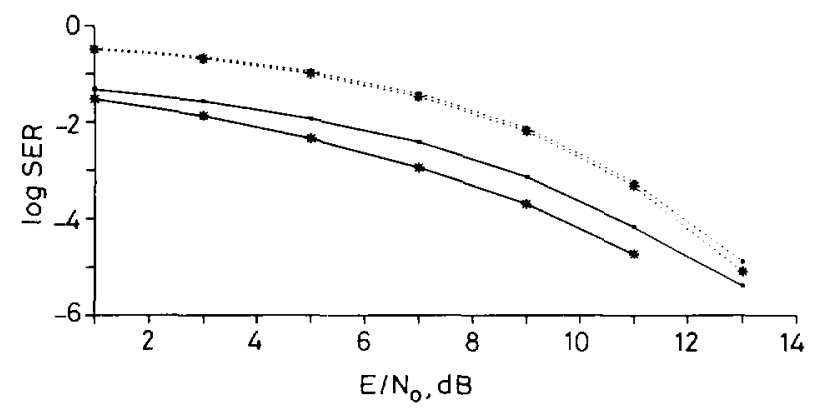

Fig. 7 CCMA decoding schemes users sink SER (code 2)

- - User 1 HD_CCMA

- - User 1 MLSD_CCMA

User 2 HD_CCMA

User 2 MLSD_CCMA

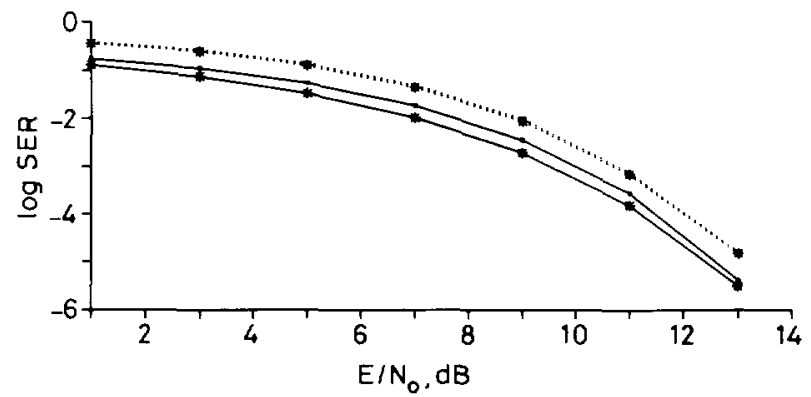

Fig. 8 CCMA decoding schemes users sink SER (code 3)

- - User 1 HD_CCMA

- - User 1 MLSD_CCMA

User 2 HD_CCMA

User 2 MLSD_CCMA

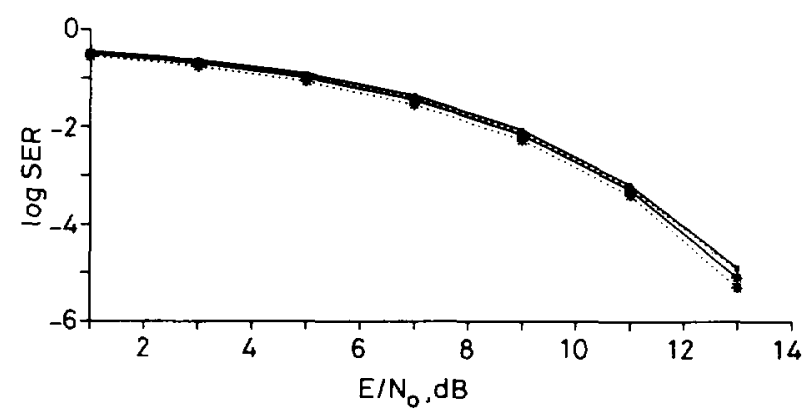

Fig. 9 CCMA decoding schemes users sink SER (code 4)

$$
\begin{aligned}
& \begin{array}{l}
- \text { - User } 1 \text { HD_CCMA } \\
\text { - - User } 1 \text { MLSD_CCMA }
\end{array} \\
& \text { User } 2 \text { HD_CCMA } \\
& \text { User } 2 \text { MLSD_CCMA }
\end{aligned}
$$

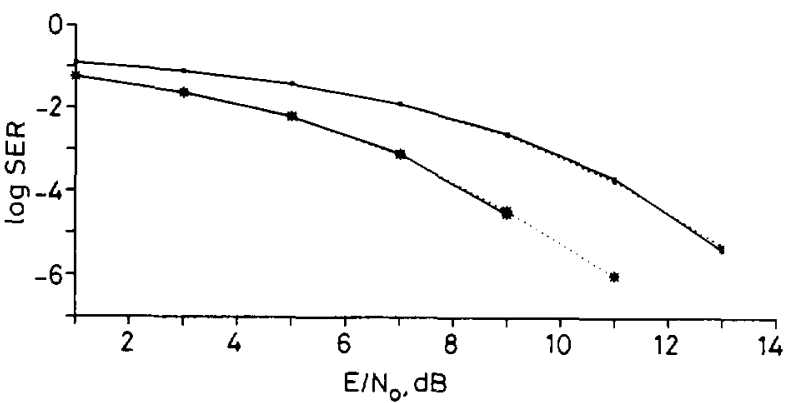

Fig. 10 CCMA decoding schemes users sink SER (code 5)

$$
\begin{aligned}
& \text { - User } 1 \text { HD_CCMA } \\
& \text { - * User } 1 \text { MLSD_CCMA } \\
& \cdots \text { User } 2 \text { HD_CCMA } \\
& \text {.. * User } 2 \text { MLSD_CCMA }
\end{aligned}
$$

more than $2.5 \mathrm{~dB}$ at $10^{-6}$ error probability is achievable employing MLSD_CCMA over the HD_CCMA decoding technique.

\section{Conclusion}

Collaborative coding and decoding techniques to utilise the MAC function and error control capability have been described. In particular, HD_CCMA and MLSD_CCMA decoding techniques were described in conjunction with SBS_HD decoding. A low complexity MLSD decoding technique is introduced. Reliability performance evaluation has been carried out with various collaborative codes.

It has been shown that uniquely decodable CCMA schemes permit the multiple access function to be combined with that of forward error protection assuming symbol and block synchronisations are maintained. The MLSD_CCMA decoding technique decreases the overall probability of error with some energy gain. The energy gain achieved is higher when the codes used have some error protection capability. A coding gain of more than $2.5 \mathrm{~dB}$ has been achieved employing MLSD_CCMA over HD_CCMA decoding technique.

Although the analyses in this paper have been carried out specifically for given two-user codes, generally the gain is achievable for any $T$-user coding scheme with error correction capability and a low complexity decoding technique which utilises this correction power. In addition, the synchronisation requirements should be reduced to a minimum, and, ideally, the system would be completely asynchronous.

\section{References}

1 SHANNON, C.E.: 'Two-way communication channels'. Proceedings of 4th Berkeley Symposium Math. Statist. and Prob., Vol. 1, pp. 611-644, 1961. Reprinted in SLEPIAN, D. (Ed.): 'Key papers in the development of information theory' (IEEE Press, New York, 1974), pp. 339-372

2 AHLSWEDE, R.: 'Multi-way communication channels'. Proceedings of 2nd International Symposium Information Theory, Tsahkadsor, Armenian, USSR, 1971 (Publishing House of the Hungarian Academy of Sciences, 1973), pp. 23-52

3 LIAO, HENRY, H.J.: 'Multiple access channels'. PhD dissertation, Department of Elect. Engineering, University of Hawaii, Honolulu, HI, 1972

4 MEULEN VAN DER, E.C.: 'A survey of multi-way channels in information theory: 1961-1976', IEEE Trans., 1977, IT-23, (1), pp. 1-37

5 MEULEN VAN DER, E.C.: 'Capacity limits for multiple access channels'. NATO Advanced Study Institute on 'Performance limits in communications theory and practice'. Ciocco, Italy, 1986

6 EL GAMAL, A., and COVER, T.M.: 'Multiple user information theory', Proc. IEEE, 1980, 68, (12), pp. 1466-1483

7 HONARY, B., and ALI, F.H.: 'Capacity of T-user collaborative coding multiple access scheme operating over a noisy channel', Electron. Lett., 1989, 25, (11), pp. 742-744

8 KASAMI, T., LIN, S., and YAMAMURA, S.: 'Further results on coding for a multiple access channel'. Conference Proceedings, Hungarian Colloquium on Information Theory, Keszthely, 1975, pp. 369-391

9 KASAMI, T., and LIN, S.: 'Coding for a multiple-access channel', IEEE Trans., 1976, IT-22, (2), pp. 129-137

10 KASAMI, T., and LIN, S.: 'Bounds on the achievable rate of block coding for a memoryless multiple-access channel', IEEE Trans., 1978, IT-24, pp. 187-197

11 FARRELL, P.G.: 'Survey of channel coding for multi user systems', in SKWIRZYNSKI, J.K. (Ed.): 'New concept in multiuser communication' (Sijthoff and Noordhoff, 1981), pp. 133-159

12 WOLF, J.K.: 'Constructive codes for multi-user communication channels', in LONGO, G. (Ed.): Part 2 (Shannon Theory) of 'Information theory: new trends and open problems'. CISM Courses and Lectures No. 219 (Springer-Verlag, 1975) 
13 WOLF, J.K.: 'Coding techniques for multiple access communication channels', in SKWIRZYNSKI, J.K. (Ed.): 'New concept in multiuser communication' (Sijthoff and Noordhoff, 1981), pp. 83-103

14 WELDON, E.J. Jr.: 'Coding for a multiple-access channels', Information and Control, 1978, 36, pp. 256-274

15 CHANG, S.C., and WELDON, E.J.: 'Coding for T-user multipleaccess channels', IEEE Trans., 1979, IT-25, (6), pp. 684-691

16 CHANG, S.C., and WOLF, J.K.: 'On the T-user M-frequency noiseless multiple access channel with and without intensity information' IEEE Trans., 1981, IT-27, (1), pp. 41-48

17 CHANG, S.C.: 'Further results on coding for T-user multiple access channels', IEEE Trans., 1984, IT-30, (2), pp. 411-415

18 KHACHATRIAN, G.H.: 'New construction of linear $\delta$-decodable codes for 2-user adder channel', Prob. Contr. Inform. Theory, 1984 13, pp. 275-279
19 BRAAK VAN DEN COEBERGH, P.A., and TILBORG VAN DEN, H.C.: 'A family of good uniquely decodable pairs for the twoaccess binary channel', IEEE Trans., 1985, IT-31, (1), pp. 3-9

20 WILSON, J.: 'Error correcting codes for a T-user binary adder channel', IEEE Trans., 1988, IT-34, (4), pp. 888-890

21 MATHYS, P.: 'Multiple access coding with error control: a code construction for the real adder channel'. Proceedings of 4th Swedish-Soviet International Workshop on Information Theory, Gotland, Sweden, 27th August-1st September 1989, pp. 175-179

22 ALI, F.H., and HONARY, B.: 'Low complexity soft decision decoding technique for $T$-user collaborative coding multiple-access channels', Electron. Lett., 1991, 27, (13), pp. 1167-1169 